\title{
Specimen Preparation for X-ray Micro-Computed Tomography of Plants
}

\author{
E.E. Johnson, D.M. Dwarte, and A.S. Jones
}

Australian Centre for Microscopy \& Microanalysis, The University of Sydney, Sydney, NSW Australia 2006

The use of X-ray micro-computed tomography (micro-CT) to produce high-resolution threedimensional reconstructions of biological specimens is becoming increasingly widespread. The technique is most commonly applied to bone and dental imaging [1], and is also being used in cancer research [2]. With regards to plants, the non-invasive nature of micro-CT is perfect for visualizing delicate anatomical structures (eg: embryos, the shoot apical meristem and infloresences) without tissue dissection. However, the application of micro-CT to plant research has been largely limited to woody tissue $[3,4]$, with only a handful of studies focusing on soft plant tissue $[5,6]$. As a result, specific specimen preparation techniques for micro-CT of soft plant tissue are not well established.

While plant specimens may be scanned fresh without special preparation [5], there are two main factors affecting the success of the scan: 1) dessication, causing movement over the course of the scan and 2) transparency to X-rays, resulting in low contrast. Indeed, for a high resolution reconstruction, the step size between each scan should be less than $0.5^{\circ}$, which, together with the relatively long exposure times required for unstained plant material, means scan times can take upwards of several hours for optimal results (Fig 1). Dessication of soft tissue over this time can be difficult to prevent, but may be avoided/reduced, by constructing an X-ray transparent humid chamber (eg: a sealed drinking straw) for the sample to be scanned in or by coating the plant tissue in vaseline, although the latter technique may reduce image resolution [7].

We have investigated how well specimen preparation techniques used for electron microscopy can be applied to high-resolution micro-CT of plants. Arabidopsis seedlings were chemically fixed, osmicated, dehydrated and critical point dried before being scanned in the micro-CT (Xradia 400). Seedlings remained stable during the $16 \mathrm{hr}$ scan, while the heavy metal treatment substantially increased contrast, particularly of the apical meristem (Fig. 2). We are also exploring the usefulness of other stains commonly used in plant electron microscopy (eg: tannic acid) across a range of different plant specimens. An additional advantage of preparing samples in this way is the potential applicability to nano-CT, which has strict sample thickness conditions and involves extremely long scan times to achieve sub- $0.1 \mu \mathrm{m}$ resolution. We are currently optimizing techniques to achieve subcellular resolution of plant specimens and will also present these novel results here [8].

References

[1] G.R. Davis \& F.S Wong, Physiol. Meas. 17(2) (1996) 121.

[2] M.J. Paulus et al., Neoplasia. 2(1-2) (2000) 62.

[3] S.C. Mayo et al., J. Struct. Biol. 171(2) (2010) 182.

[4] K. Steppe et al., J. Struct. Biol. 148(1) (2004) 11.

[5] W. Stuppy et al., Trends Plant Sci. 8(1) (2003) 2.

[6] S. Dhondt et al., Trends Plant Sci. 15(8) (2010) 419.

[7] N. Korte \& S. Porembski, Microsc. Res Tech. (2010) DOI 10.1002/jemt.20917.

[8] Arabidopsis material was kindly supplied by Dr Brian Jones (University of Sydney). 

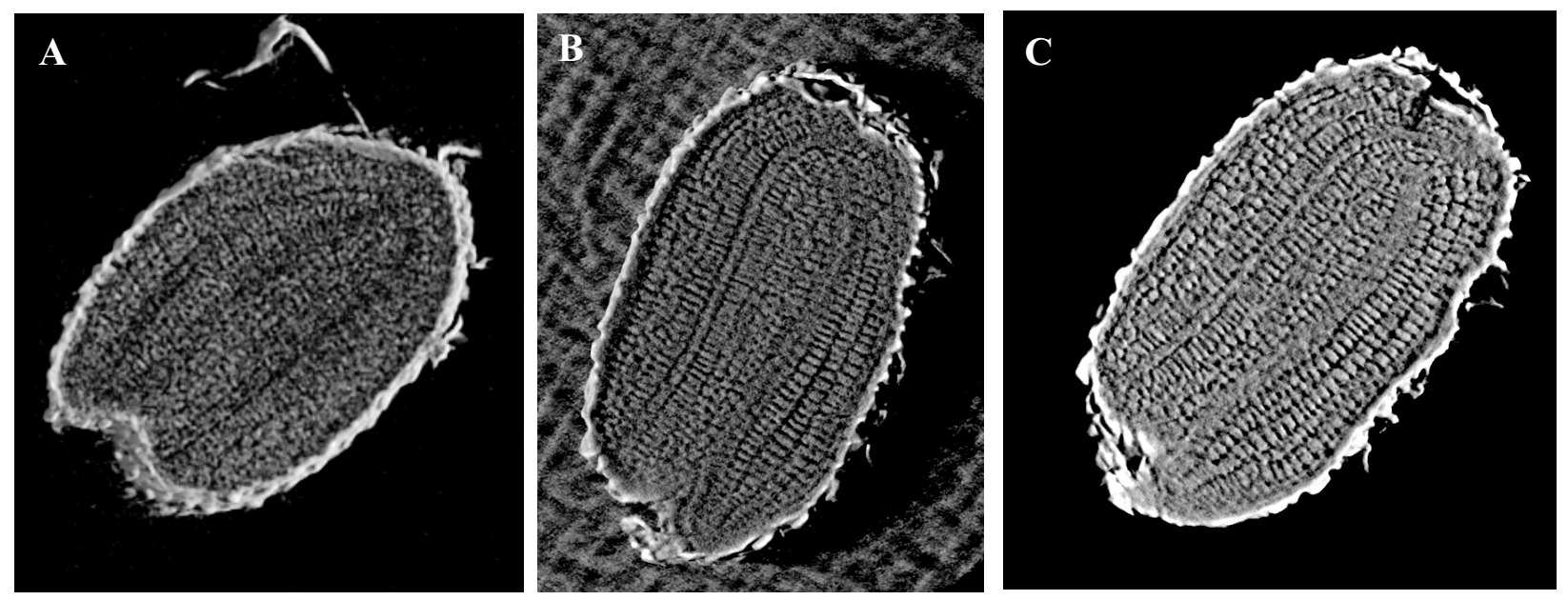

FIG 1. Three-dimensional cutaway reconstructions of untreated Arabidopsis seeds using different scanning parameters. A: Low resolution 80 min scan at 10x magnification, with 365 images and $5 \mathrm{sec}$ exposure; B: Mid-resolution $4 \mathrm{hr}$ scan at 20x magnification, with 365 images and $20 \mathrm{sec}$ exposure; C: Higher resolution $12 \mathrm{hr}$ scan at 20x magnification, with 729 images and $30 \mathrm{sec}$ exposure, which yielded the best cellular resolution of the developing embryo. Scans were performed on an Xradia microXCT-400 at $60 \mathrm{kV}$.
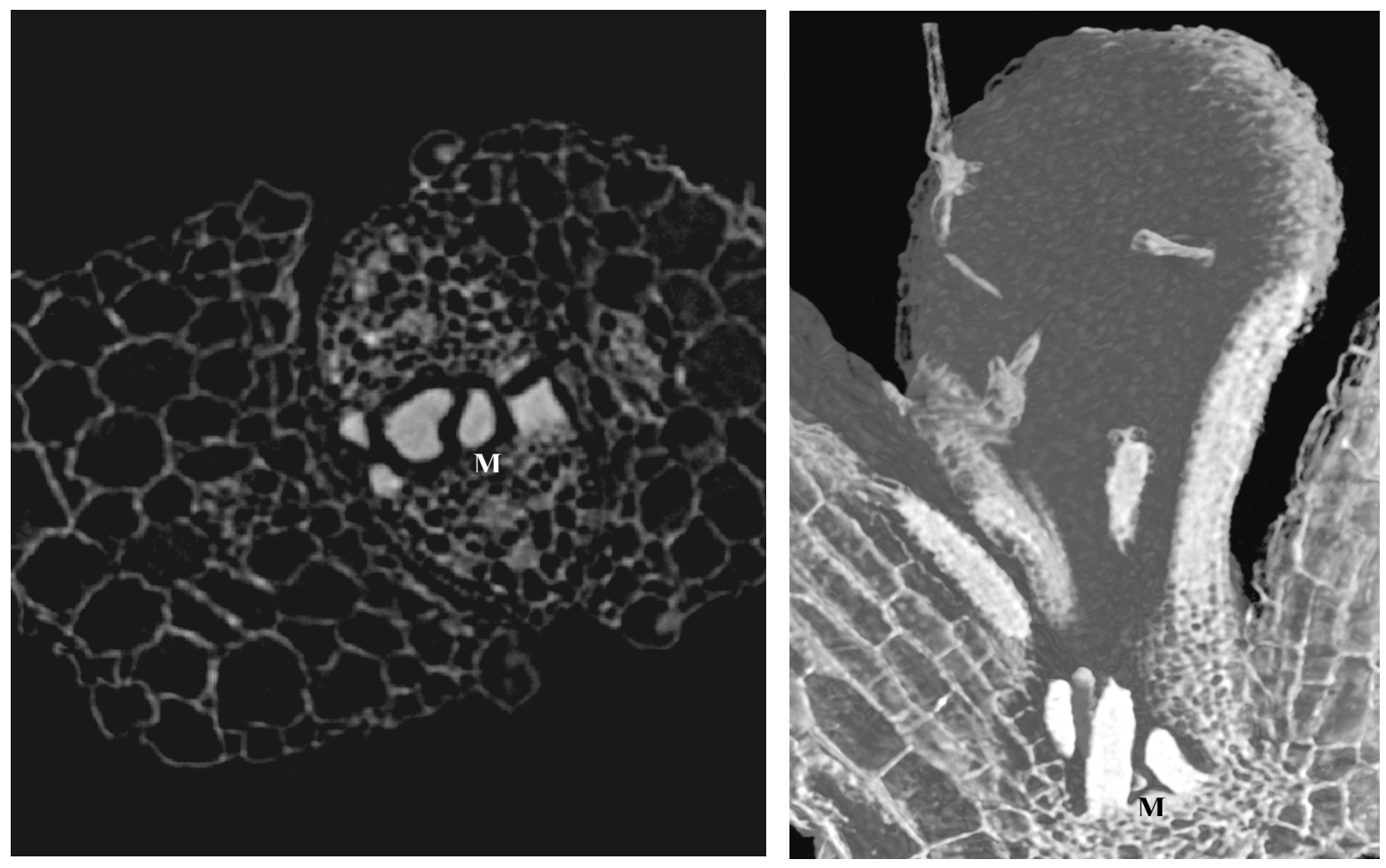

FIG 2. Axial slice (left) and three-dimensional cutaway reconstruction (right) of an Arabidopsis seedling prepared as for SEM. The seedling was scanned for $16 \mathrm{hrs}$ at $20 \mathrm{x}$ magnification, with 911 images and $50 \mathrm{sec}$ exposure, on the Xradia microXCT-400 at 80 $\mathrm{kV}$. The shoot apical meristem $(\mathrm{M})$ shows relatively high contrast. 